\title{
Distributed Optimization for Mobile Robots under Mobile Edge Computing Environment
}

\author{
Hui Luo $\mathbb{D}$ and Quan Yin \\ School of Artificial Intelligent and Automation, Huazhong University of Science and Technology, Wuhan, China \\ Correspondence should be addressed to Hui Luo; keyluo@hust.edu.cn
}

Received 3 June 2021; Accepted 3 September 2021; Published 21 October 2021

Academic Editor: Xiao Ling Wang

Copyright (c) 2021 Hui Luo and Quan Yin. This is an open access article distributed under the Creative Commons Attribution License, which permits unrestricted use, distribution, and reproduction in any medium, provided the original work is properly cited.

\begin{abstract}
Driven by the development of the Internet industry, mobile robots (MRs) technology has become increasingly mature and widely used in all walks of life. Since MRs are densely distributed in the network system, how to establish a reliable communication architecture to achieve good cooperation and resource sharing between MRs has become a research hotspot. In this respect, mobile edge computing (MEC) technology and millimeter wave $(\mathrm{mmW})$ technology can provide powerful support. This paper proposes a $\mathrm{mmW}$ communication network architecture for distributed MRs in MEC environment. The mmW base station provides reliable communication services for MRs under the coverage of information cloud (IC). We design a joint resource and power allocation strategy aimed at minimizing network energy consumption. First, we use the Lyapunov optimization technique to transform the original infinite horizon Markov decision process (MDP) problem. Then, a semidistributed algorithm is introduced to solve the distributed optimization problem in the mmW network. By improving the autonomous decision-making ability of the $\mathrm{mmW}$ base station, the signaling overheads caused by information interaction are reduced, and information leakage is effectively avoided. Finally, the global optimal solution is obtained. Simulation results demonstrate the superiority of the proposed strategy.
\end{abstract}

\section{Introduction}

With the rapid development of the Internet industry, a variety of intelligent terminals emerge in an endless stream. Among them, intelligent MRs can set environmental perception, dynamic decision-making and planning, behavior control and execution, and other functions in one, widely used in the industry, agriculture, medical, service, and other industries, and has aroused universal attention all over the world. In order to realize good cooperation and resource sharing among multiple MRs, so that they can assist or replace human work to the greatest extent, we need to establish a communication framework among MRs. In the current research, the multirobot wireless network system can realize flexible cooperation through a central server [1]. Based on TCP and UDP in the local area network (LAN), the distributed communication method proposed in [2] allows robots to subscribe to target topics independently to achieve multirobot cross-platform communication. Each robot in [3] can establish communication paths to maintain a consistent communication with the cloud to effectively realize cooperative communication and link prediction. The authors in [4] proposed a scheme of mobile robot object recognition in Internet of Things (IOT) and used edge nodes to coordinate the data from robot vision.

In the traditional distributed communication network architecture, a centralized management scheme centered on the core network is usually adopted. The core network collects global network information and makes global decisions. With the gradual expansion and complexity of network structure, centralized management scheme will inevitably lead to huge signaling overheads, time delay, and privacy leakage. Among the existing distributed algorithms, the authors in $[5,6]$ proposed a distributed downlink resource allocation and multidomain interference management algorithm to alleviate the co-channel interference in 
small cellular networks and maximize the total utility of the whole system. The authors in [7] proposed a distributed algorithm based on the machine learning framework of echo state networks, aiming at the problems of user association and spectrum allocation in heterogeneous small cellular networks, which allowed small base stations to independently complete the optimal allocation of resources under the condition of limited system state information and greatly reduced the wireless network information exchange. The authors in [8] proposed an adaptive semidistributed algorithm that can jointly allocate power and spectrum resources on licensed and unlicensed bands, which can improve the independent adaptability of the base station and maximize the global spectrum efficiency. Based on the research above, we need to develop a distributed network resource management scheme which is more suitable for MRs network and has stronger autonomy and flexibility, higher security, and cost performance.

In this regard, Mobile Edge Computing (MEC) technology can sink elastic resources such as computing, network, and storage in cloud data center to the edge of distributed network, effectively relieving the pressure on core network, improving computing efficiency, and reducing service latency [9-11]. MEC deeply integrates mobile access network with Internet service, which is very suitable for the MRs communication system with distributed structure. Meanwhile, to meet the development needs of high speed, low delay, and large capacity of mobile communication network in the future, human beings are exploring more abundant spectrum resources in higher frequency band. Among them, millimeter wave ( $\mathrm{mmW}$ ) not only has a wide spectrum, which can greatly improve the channel capacity, but also has a good direction, which can effectively reduce channel interference and protect user privacy [12]. Applying $\mathrm{mmW}$ technology to MRs communication networks can further improve network performance and user experience, which is an important trend of distributed MRs network development. In the existing work, the authors in [13] combined the computational shunt technology in MEC with $\mathrm{mmW}$ communication to conduct joint optimization of computing and communication resources, forming an energy-saving and efficient computing system. Aiming at distributed small cellular networks, a green load management and resource allocation scheme based on MEC is proposed in $[14,15]$, which is constrained by network cost and communication quality to achieve green computing shunt and reduce the total energy consumption of users to the greatest extent.

Based on the current research, we propose a distributed MRs communication network architecture in MEC environment. The $\mathrm{mmW}$ access points (APs) are randomly distributed under the coverage of the information cloud (IC) to provide communication services for the densely deployed MRs, in which the IC is responsible for information exchange, storage, and interaction. To minimize the energy consumption of the MRs network and achieve the optimal network performance, we designed a joint resource and power allocation strategy based on MEC under the condition of guaranteeing the network traffic delay, limiting rate, interference, and power. First, we use the Lyapunov optimization technique to transform the infinite horizon problem in the objective function into a single frame problem that can be processed. Second, we introduce a semidistributed algorithm for distributed optimization issues on complex mmW MECs. Compared with traditional distributed programs, this scheme improves the independent decision-making capacity of mmW APs and only needs to exchange a small amount of Lagrangian parameters through IC without sharing global information, which greatly reduces signaling overheads and protects the users' privacy.

The rest of the paper is organized as follows. In Section 2, we describe the system model of the distributed MRs communication network. Section 3 formulates and transforms the optimization problem. The semidistributed resource and power allocation are proposed in Section 4. Section 5 shows the simulation evaluation, and we draw the conclusion in Section 6.

Notations: $\|\cdot\|$ is the Euclidean norm. Let $\operatorname{diag}\left(x_{1}, \ldots, x_{K}\right)$ be the diagonal matrix. I denotes the identity matrix. $\mathbf{A} \otimes \mathbf{B}$ means the Kronecker product of matrixes $\mathbf{A}$ and $\mathbf{B}$. $\mathbb{E}[\cdot]$ represents the statistical expectation.

\section{System Model}

In the system, under the coverage of the IC that introduces MEC technology and is responsible for data calculation, storage, and interaction, there are I mmW APs and each of them provides services to $J$ MRs within its coverage as shown in Figure 1. The set of $\mathrm{mmW}$ APs and MRs is denoted as $\mathscr{I}=\left\{W_{1}, W_{2}, \ldots, W_{I}\right\}$ and $\mathscr{J}=\left\{R_{1}, R_{2}, \ldots, R_{J}\right\}$. Each $\mathrm{mmW}$ AP equips with mmW transceivers to overcome the significantly high path loss though highly directional transmission, and each MR introduces MEC technology and equips with a $\mathrm{mmW}$ beam steerable array to support transmission over IC and mmW network.

We have each MR implementing the analog beamforming, allowing one transmission link each time, and each $\mathrm{mmW}$ AP implementing the hybrid beamforming, allowing multiple transmission links each time. The mmW AP $W_{i}$ equipped with $C_{i}$ radio frequency $(\mathrm{RF})$ chains can serve most $C_{i}$ MRs. Moreover, we assume that the proposed network transmits in a slotted system, which is represented as $t=\{0$, $1,2, \ldots\}$. Denote $x_{i, j}(t)=1$ if the $j$-th MR is in the coverage of the mmW AP $W_{i}$ at time slot $t$, and $x_{i, j}(t)=0$ otherwise. We assume each MR is attached to not more than one $\mathrm{mmW}$ $\mathrm{AP}$, i.e., $\sum_{i=1}^{I} x_{i, j}(t) \leq 1, \forall j \in \mathcal{J}$. We further assume that the total number of MRs attached to the mmW AP does not exceed the maximum chain number, i.e., $\sum_{j=1}^{J} x_{i, j}(t) \leq C_{i}, \forall i \in \mathscr{I}$.

2.1. mmW Transmission Model. The mmW network is susceptible to interference and has severe channel attenuation. By applying beamforming technology, directional transmission can be formed between mmW APs and MRs to increase data rate. According to [16], we build the model of $\mathrm{mmW}$ network transmission as shown in Figure 2. Let $\theta_{i}^{a}(t)$ 


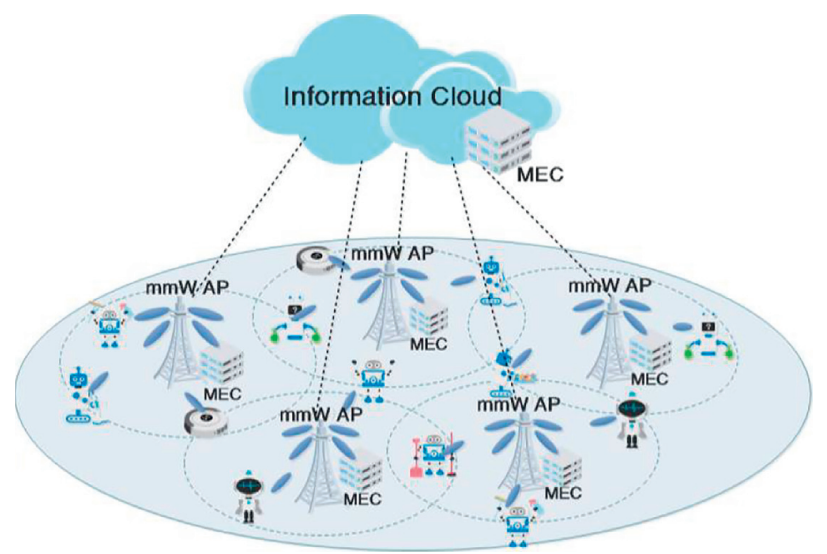

FIgURE 1: The network framework for mobile robots in MEC environment.

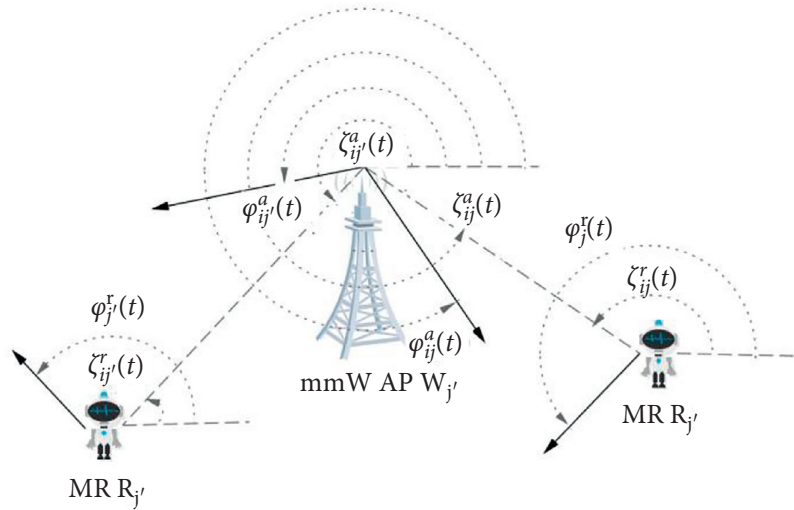

FIgURE 2: Illustration of the angles between the mmW AP and MRs in the transmission.

and $\theta_{j}^{r}(t)$ be the operating beamwidths of mmW AP $W_{i}$ and MR $R_{j}$, respectively. Let $\zeta_{i j}^{a}(t)$ be the angle between the positive $x$-axis and the direction in which the $\mathrm{mmW}$ AP $W_{i}$ sees MR $R_{j}$, and let $\zeta_{i j}^{r}(t)$ be similarly defined by changing the roles of the mmW AP $W_{i}$ and MR $R_{j}$. The values of $\zeta_{i j}^{a}(t)$ and $\zeta_{i j}^{r}(t)$ satisfy $\left|\zeta_{i j}^{a}(t)-\zeta_{i j}^{r}(t)\right|=\pi$. We further denote $\varphi_{i j}^{a}(t)$ and $\varphi_{j}^{\mathrm{r}}(t)$ as the boresight angles of the mmW AP $W_{i}$ and MR $R_{j}$ relative to the positive $x$-axis. Let $g_{i j, j i}^{a}(t)$ be the transmission gain between the beam from the mmW AP $W_{i}$ to MR $R_{j .}$ and $g_{j i, i j}^{r}(t)$ be the reception gain between the beam from MR $R_{j}$ to the mmW AP $W_{i}$, which can be written as follows.

$$
\begin{aligned}
& g_{i j, j i}^{a}(t)=\left\{\begin{array}{cc}
\varepsilon, & \text { if } \frac{\theta_{i}^{a}(t)}{2}<\left|\varphi_{i j}^{a}(t)-\zeta_{i j}^{a}(t)\right|<2 \pi-\frac{\theta_{i}^{a}(t)}{2}, \\
\frac{2 \pi-\left(2 \pi-\theta_{i}^{a}(t)\right) \varepsilon}{\theta_{i}^{a}(t)}, & \text { otherwise, } \\
\varepsilon, & \text { if } \frac{\theta_{i}^{r}(t)}{2}<\left|\varphi_{j}^{r}(t)-\zeta_{i j}^{r}(t)\right|<2 \pi-\frac{\theta_{i}^{r}(t)}{2}, \\
g_{j i, i j}^{r}(t)=\left\{\begin{array}{cc}
2 \pi-\left(2 \pi-\theta_{i}^{r}(t)\right) \varepsilon \\
\theta_{i}^{r}(t)
\end{array}\right. & \text { otherwise. }
\end{array}\right\}
\end{aligned}
$$

2.2. Interference Management. According to [16], there are two types of interference in the proposed $\mathrm{mmW}$ network that should be managed. The intracell interference exists among devices within a cell and can be mitigated by using proper scheduling and beamforming design. The intercell interference exists among different cells and can be 
significantly reduced by resource blocks scheduling and omnidirectional communication. Therefore, to ensure that the intercell interference in the $\mathrm{mmW}$ network is small enough to be ignored, the following constraints can be introduced:

$$
\sum_{\substack{i^{\prime} \in \mathcal{I} \\ i^{\prime} \neq i}} \sum_{\substack{j^{\prime} \in \mathcal{F} \\ j^{\prime} \neq j}} p_{i^{\prime} j^{\prime}}^{a}(t) g_{i^{\prime} j^{\prime}, j i}^{a}(t) g_{i^{\prime} j}^{c}(t) g_{j^{\prime} i^{\prime}, i j}^{r}(t) \leq I^{T},
$$

where $I^{T}$ is the intercell interference threshold, $p_{i j}^{a}(t)$ denotes the transmit power from the mmW AP $W_{i}$ to $\mathrm{MR} R_{j}$, and $g_{i j}^{c}(t)$ refers the channel gain between the mmW AP $W_{i}$ to MR $R_{j}$.
2.3. Data Transmission. Let $Q_{j}(t)$ be the data queue length of MR $R_{j}$ at time slot $t$. The data entering the queue of MR $R_{j}$ at time slot $t$ are denoted as $A_{j}(t)$, whose maximum value is $A_{j \text {, max }}(t)$. The traffic buffer queue $Q_{j}(t)$ of MR $R_{j}$ evolves as

$$
Q_{j}(t+1)=\left[Q_{j}(t)-R_{j}^{a}(t) \tau\right]^{+}+A_{j}(t) \tau, \quad \forall j \in \mathscr{J}, t,
$$

where $[x]^{+}=\max \{x, 0\}$ is on account that the actual served packets cannot be larger than the current queue size. $\tau$ is the duration of each time slot. We further let $Q_{j}(0)=0, \forall j \in \mathcal{J}$ for simplicity.

The achievable data rate of MR $R_{j}$ served by the $\mathrm{mmW}$ AP $W_{i}$ at time slot $t$ can be written as

$$
R_{i j}^{a}(t)=x_{i, j}(t) B^{a} \log _{2}\left(1+\frac{p_{i j}^{a}(t) g_{i j, j i}^{a}(t) g_{i j}^{c}(t) g_{j i, i j}^{r}(t)}{B^{a} N_{0}}\right), \quad \forall j \in \mathcal{J}, i \in \mathcal{I}, t,
$$

where $B^{a}$ is the mmW bandwidth and $N_{0}$ is the noise power spectral density. Based on (4), the achievable data rate of MR $R_{j}$ served by the mmW network at time slot $t$ is given by

$$
R_{j}^{a}(t)=\sum_{i=1}^{I} R_{i j}^{a}(t), \quad \forall j \in \mathscr{J}, t .
$$

\section{Problem Formulation and Transformation}

3.1. Problem Formulation. Our goal in this work is to design a resource and power allocation strategy to minimize the energy consumption of devices and guarantee power and rate limitation and traffic latency of MRs as well as ensuring performance of the mmW network. The energy aware optimization problem can be formulated as

$$
\text { P1: } \min _{\left\{\mathbf{p}^{a}\right\}} \limsup _{T \longrightarrow+\infty} \frac{1}{T} \sum_{t=0}^{T-1} \mathbb{E}\left[\sum_{j \in \mathcal{F}} p_{j}^{a}(t) \tau\right]
$$

subject to (2),

$$
\begin{aligned}
& \underset{T \longrightarrow+\infty}{\limsup } \frac{1}{T} \sum_{t=0}^{T-1} \mathbb{E}\left[Q_{j}(t)\right]<\bar{Q}_{j}, \forall j \in \mathscr{J}, t, \\
& R_{j}^{a}(t) \geq R_{j}^{T}, \forall j \in \mathcal{J}, t, \\
& p_{j}^{a}(t) \leq P_{j}^{T}, \quad \forall j \in \mathcal{J}, t, \\
& p_{i j}^{a}(t) \geq 0, \quad \forall j \in \mathscr{J}, i \in \mathscr{I}, t,
\end{aligned}
$$

where $p_{j}^{a}(t)=\sum_{i \in \mathscr{I}} p_{i j}^{a}(t), \bar{Q}_{j}$ is the predefined average queue backlog tolerance of MR $R_{j}$, and $R_{j}^{T}(t)$ and $P_{j}^{T}$ are the minimum data rate requirement and the maximum power consumption of MR $R_{j}$ at time slot $t$. Constraint (7a) ensures the time-averaged latency of $M R$, which is proportional to the average queue backlog length according to Little's theorem. Constraint $(7 \mathrm{~d})$ is the limitation of parameters.
3.2. Lyapunov Drift Penalty. Since the optimization function $\mathrm{P} 1$ is a long-term average network energy consumption problem, it is an infinite horizon Markov decision process (MDP) problem [17]. To tackle the infinite horizon problem P1 with lower computational complexity and less memory consumption, we introduce the Lyapunov drift penalty technique to transform $\mathrm{P} 1$ into a tractable single frame problem [18]. We define a virtual queue $Z_{n}$ for constraint (7a) as

$$
Z_{j}(t+1)=\left[Z_{j}(t)+Q_{j}(t+1)-\bar{Q}_{j}\right]^{+}, \quad \forall j \in \mathcal{J}, t .
$$

For each slot $t$, the Lyapunov function can be formulated as a sum of virtual queue length squares, given by

$$
L(\mathbf{Z}(t))=\frac{1}{2}\left(\sum_{j=1}^{J} Z_{j}^{2}(t)\right), \quad \forall t
$$

where $\mathbf{Z}(t)=\left[Z_{1}(t), Z_{2}(t), \ldots, Z_{J}(t)\right]$.

Moreover, the Lyapunov drift function is expressed as

$$
\triangle \mathbf{Z}(t)=\mathbb{E}[L(\mathbf{Z}(t+1))-L(\mathbf{Z}(t)) \mid \mathbf{Z}(t)], \quad \forall t .
$$

Then, the Lyapunov drift penalty function is defined to minimize the drift-plus-penalty expression boundary, which can be formulated as

$$
\begin{aligned}
\Delta_{v} \mathbf{Z}(t)= & \mathbb{E}\left[\frac{1}{2} \sum_{j \in \mathcal{F}}\left(Z_{j}^{2}(t+1)-Z_{j}^{2}(t)\right) \mid \mathbf{Z}(t)\right] \\
& +V \mathbb{E}\left[\sum_{j \in \mathcal{F}} p_{j}^{a}(t) \tau \mid \mathbf{Z}(t)\right], \quad \forall t,
\end{aligned}
$$

where $V$ is the nonnegative control parameter, which represents the weight of the objective function.

Lemma 1. The upper bound for the Lyapunov drift penalty (11) can be written as 


$$
\begin{aligned}
\triangle_{\nu} \mathbf{Z}(t) \leq & \sum_{j \in \mathcal{F}} \frac{Z_{j 1}+Z_{j 2}}{2}+V \mathbb{E}\left[\sum_{j \in \mathcal{F}} p_{j}^{a}(t) \tau \mid \mathbf{Z}(t)\right] \\
& -\mathbb{E}\left[\sum_{j \in \mathcal{F}}\left(Q_{j}(t)+Z_{j}(t)\right) R_{j}^{a}(t) \tau \mid \mathbf{Z}(t)\right], \quad \forall t .
\end{aligned}
$$

Here, $Z_{j 1}=\left(Q_{j, \max }+A_{j, \max }\right)^{2}+\left(Q_{j, \max }\right)^{2}+\left(\bar{Q}_{j}\right)^{2}, Z_{j 2}$ $=2 Z_{j, \text { max }}\left(Q_{j, \text { max }}+A_{j, \max }\right)$, and $Q_{j, \text { max }}$ and $Z_{j, \text { max }}$ are upper bounds for $Q_{j}(t)$ and $Z_{j}(t)$.

Proof. See Appendix A.

From Lemma 1, P1 can be decoupled into the following auxiliary problem at each single SP $t$ :

$$
\text { P2: } \min _{\left\{\mathbf{p}^{a}\right\}} V \sum_{j \in \mathscr{f}} p_{j}^{a}(t)-\sum_{j \in \mathscr{F}}\left(Q_{j}(t)+Z_{j}(t)\right) R_{j}^{a}(t),
$$

subject to (2) and (7b)-(7d). For more precise transformation, we introduce Theorem 1 to prove the relationship between the solutions of $\mathrm{P} 1$ and $\mathrm{P} 2$.

Theorem 1. $S^{\beta}$ is denoted as the optimal average energy consumption under $\mathrm{P} 2$, which is limited by an upper bound.

$$
S^{\beta} \leq \frac{\sum_{j \in \mathcal{F}}\left(Z_{j 1}+Z_{j 2}\right)}{2 V}+S^{\alpha},
$$

where $S^{\alpha}$ is the optimal average energy consumption under $\mathrm{P} 1$. The optimal value of $S^{\beta}$ can be arbitrarily close to the optimal value $S^{\alpha}$ by selecting asymptotically large $V$.

Proof. See Appendix B.

3.3. Distributed Optimization Problem. In the following, we omit the time slot index $t$ for ease of exposition. Based on P2, we introduce a block of slack variables $c=\left\{c_{1}, c_{2}, \ldots, c_{J}\right\}$ and $d=\left\{d_{1}, d_{2}, \ldots, d_{J}\right\}$ and get the decentralized $\mathrm{mmW}$ network problem as follows:

$$
\min _{\left\{\mathbf{p}^{a}\right\}} V \sum_{i \in \mathscr{I}} \sum_{j \in \mathscr{F}} p_{i j}^{a}-\sum_{i \in \mathscr{I}} \sum_{j \in \mathscr{F}}\left(Q_{i}+Z_{i}\right) R_{i j}^{a},
$$

subject to (2) and (7d),

$$
\begin{aligned}
& R_{i j}^{a} \geq x_{i, j}\left(R_{j}^{T}-c_{j}\right), \quad \forall j \in \mathscr{J}, i \in \mathscr{I}, \\
& p_{i j}^{a} \leq x_{i, j}\left(P_{j}^{T}-d_{j}\right), \quad \forall j \in \mathscr{J}, i \in \mathscr{I} .
\end{aligned}
$$

To solve the decentralized network problem, the $\mathrm{mmW}$ APs are modeled as agents which can make decisions independently. Based on the initialization-free distributed method in [17, 19], problem (15) can be rewritten as

$$
\min _{\mathbf{X}_{i}, i \in \mathscr{I}} \sum_{i \in \mathscr{I}} f_{i}\left(\mathbf{X}_{i}\right)
$$

subject to

$$
\begin{aligned}
& \sum_{i \in \mathscr{I}} \mathbf{G}_{i} \mathbf{X}_{i}=\sum_{i \in \mathscr{I}} \mathbf{I}, \\
& \mathbf{X}_{i} \in \Omega_{i}, \quad \forall i \in \mathscr{I} .
\end{aligned}
$$

where $f_{i}\left(\mathbf{X}_{i}\right)$ is a convex function which is equivalent to (15) and $\mathbf{X}_{i}=\left[p_{1 i}^{a}, \ldots, p_{J i}^{a}\right]^{T}$. Equation (18a) is the global constraint related to (2), where $\mathbf{I}=\left[I^{T}, \ldots, I^{T}\right]^{T}$, $\mathbf{G}_{i}=\left[\mathbf{g}_{i 1}, \ldots, \mathbf{g}_{i j}\right], \quad \mathbf{g}_{i j}=\left[g_{i j, 1}, \ldots, 0, \ldots, g_{i j, J}\right]^{T}, \quad$ and $g_{i j, k}=\sum_{n \in \mathscr{I}} x_{n, j} g_{i j, k n}^{a} g_{j i, n k}^{u} g_{j n}^{c}$. Equation (18b) is the local feasibility constraint of agent $i$ associated with (7c), (16a), and (16b).

The local objective function $f_{i}\left(\mathbf{X}_{i}\right)$, the interference matrix $\mathbf{G}_{i}$ and closed convex set $\Omega_{i}$ are the private information not shared with others of agent $i$. Equation (17) is a distributed optimization problem. In our framework, under the coverage of information cloud, the mmW APs can carry out data calculation and information interaction through the IC. Hence, we need to design a semidistributed joint resource and power allocation strategy for the agents, in which each agent interacts with its neighbor agents through the IC and collaborates to find its own optimal scheme.

\section{Semidistributed Resource and Power Allocation}

4.1. The Proposed Semidistributed Algorithm. First, we defined the Lagrangian duality of (17) with Lagrange multiplier $\lambda$ as

$$
\begin{aligned}
\max _{\lambda} q(\boldsymbol{\lambda}) & =\sum_{i \in \mathcal{I}} q_{i}(\boldsymbol{\lambda}) \\
& =\sum_{i \in \mathscr{Y}} \inf _{\mathbf{X}_{i} \in \Omega_{i}}\left\{f_{i}\left(\mathbf{X}_{i}\right)+\boldsymbol{\lambda}^{T}\left(\mathbf{G}_{i}-\mathbf{I}\right)\right\},
\end{aligned}
$$

where $\lambda$ can be updated via IC. Due to the global multiplier $\lambda$, we need to solve the subproblems to calculate the gradients in the IC. To avoid this shortage, we can introduce the Laplacian matrix $\mathbf{L}$ and the multiplier matrix $\Lambda=\operatorname{col}\left(\lambda_{1}, \ldots, \lambda_{I}\right)$ and reformulate the following constrained optimization problem [15].

$$
\max _{\Lambda} Q(\boldsymbol{\Lambda})=\sum_{i \in \mathscr{I}} q_{i}\left(\boldsymbol{\lambda}_{i}\right),
$$

subject to

$$
\left(\mathbf{L} \otimes \mathbf{I}_{i}\right) \boldsymbol{\Lambda}=0,
$$

where $\otimes$ denotes the Kronecker product operation and $\mathbf{I}_{i}$ is an identity matrix.

Before further defining the Laplacian matrix $\mathbf{L}$, we need to introduce the graph theory which can describe the information exchange among the agents through a graph $\mathscr{G}=(\mathcal{N}, \mathscr{E})$. According to the graph theory [20], the edge set is defined as $\mathscr{E} \subset \mathcal{N} \times \mathcal{N}$. If agent $k$ can receive information from agent $n$, then agent $n$ belongs to agent $k$ 's neighbor set, denoted as $\mathcal{N}_{k}=\{n \mid(n, k) \in \mathscr{E}\}$. In this work, we need to assume that the information exchange graph $\mathscr{G}=(\mathcal{N}, \mathscr{E})$ is 
(1) $\dot{\mathbf{X}}_{i}(\ell+1)=P_{\Omega_{i}}\left(\mathbf{X}_{i}(\ell)-\nabla f_{i}\left(\mathbf{X}_{i}(\ell)\right)+\lambda_{i}(\ell) \mathbf{G}_{i}\right)-\mathbf{X}_{i}(\ell)$;

(2) $\lambda_{i}(\ell+1)=-\sum_{k \in \mathcal{N}_{i}}\left(\lambda_{i}(\ell)-\lambda_{k}(\ell)\right)-\sum_{k \in \mathcal{N}_{k}}\left(\mathbf{z}_{i}(\ell)-\mathbf{z}_{k}(\ell)\right)+\left((\mathbf{I} / K)-\mathbf{X}_{i}(\ell) \mathbf{G}_{i}\right)$;

(3) $\dot{\mathbf{z}}_{i}(\ell+1)=\sum_{k \in \mathcal{N}_{i}}\left(\lambda_{i}(\ell)-\lambda_{k}(\ell)\right)$;

Algorithm 1: Distributed algorithm for each agent $i, i \in \mathscr{I}$.

(1) Initialize $\ell=1, \mathbf{X}(1), \Lambda(1), \mathbf{Z}(1)$

(2) Set the neighbor sets for all the mmW APs as $\mathcal{N}_{i}, \forall i \in \mathscr{I}$

(3) Let $\sigma \longrightarrow 0$

(4) while $\left(\|\dot{X}(\ell)\|_{2}^{2}+\|\dot{\Lambda}(\ell)\|_{2}^{2}+\|\dot{Z}(\ell)\|_{2}^{2}\right) \geq \sigma$

(5) $\mathrm{mmW}$ APs obtain the information of $\Lambda_{i}(\ell)$ and $\mathbf{Z}_{i}(\ell), \forall i \in \mathscr{I}$ from the information cloud;

(6) Each mmW AP $i, \forall i \in \mathscr{I}$ updates its power allocation and multiplier parameters independently based on (23);

(7) $\mathrm{mmW}$ APs $i$ feed back the information of $\Lambda_{i}(\ell)$ and $\mathbf{Z}_{i}(\ell), \forall i \in \mathscr{I}$ to the information cloud;

(8) set $\ell=\ell+1$.

(9) Endwhile

Algorithm 2: Semidistributed power allocation scheme.

TABle 1: Parameter setting.

\begin{tabular}{lc}
\hline Parameters & Values \\
\hline Minimum data rate $R^{T}$ & 0.5 to $30 \mathrm{mbps}$ \\
Maximum power consumption $P^{T}$ & $27 \mathrm{dBm}$ \\
Queue backlog tolerance $Q^{j}$ & 1 to $10 \mathrm{~ms}$ \\
Noise power spectral density $N^{0}$ & $-134 \mathrm{dBm} / \mathrm{MHz}$ \\
The mmW bandwith $B^{a}$ & $2 \mathrm{GHz}$ \\
Operating beamwidths between mmW AP and MR $\theta$ & $10^{\circ}$ \\
The intercell interference threshold $I^{T}$ & $10^{-10} \mathrm{~W}$ \\
The duration of each time slot $\tau$ & $1 \mathrm{~ms}$ \\
\hline
\end{tabular}

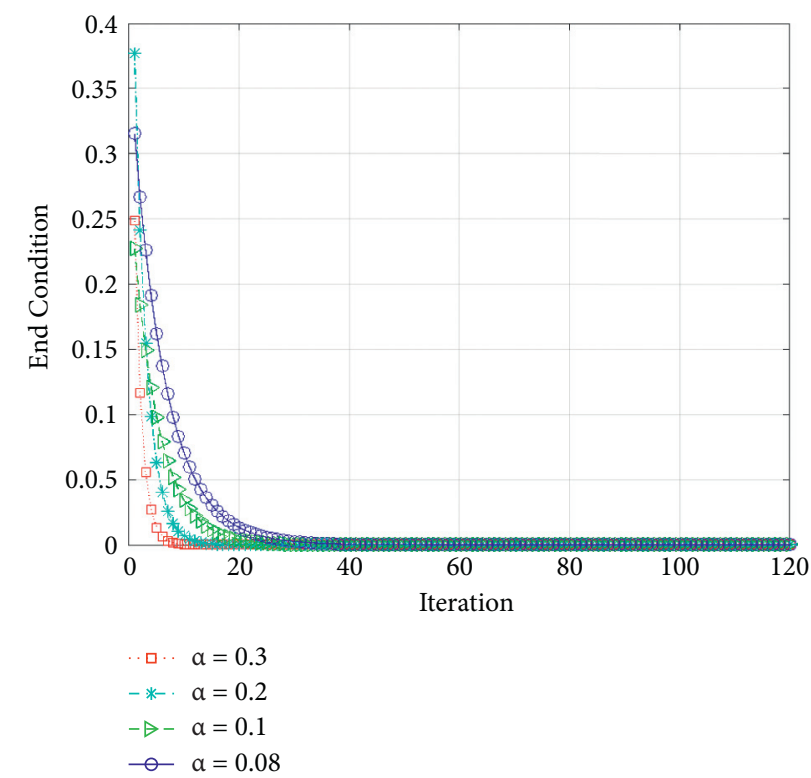

FIGURE 3: Convergence performance of Algorithm 2 under asynchronous integration step. 

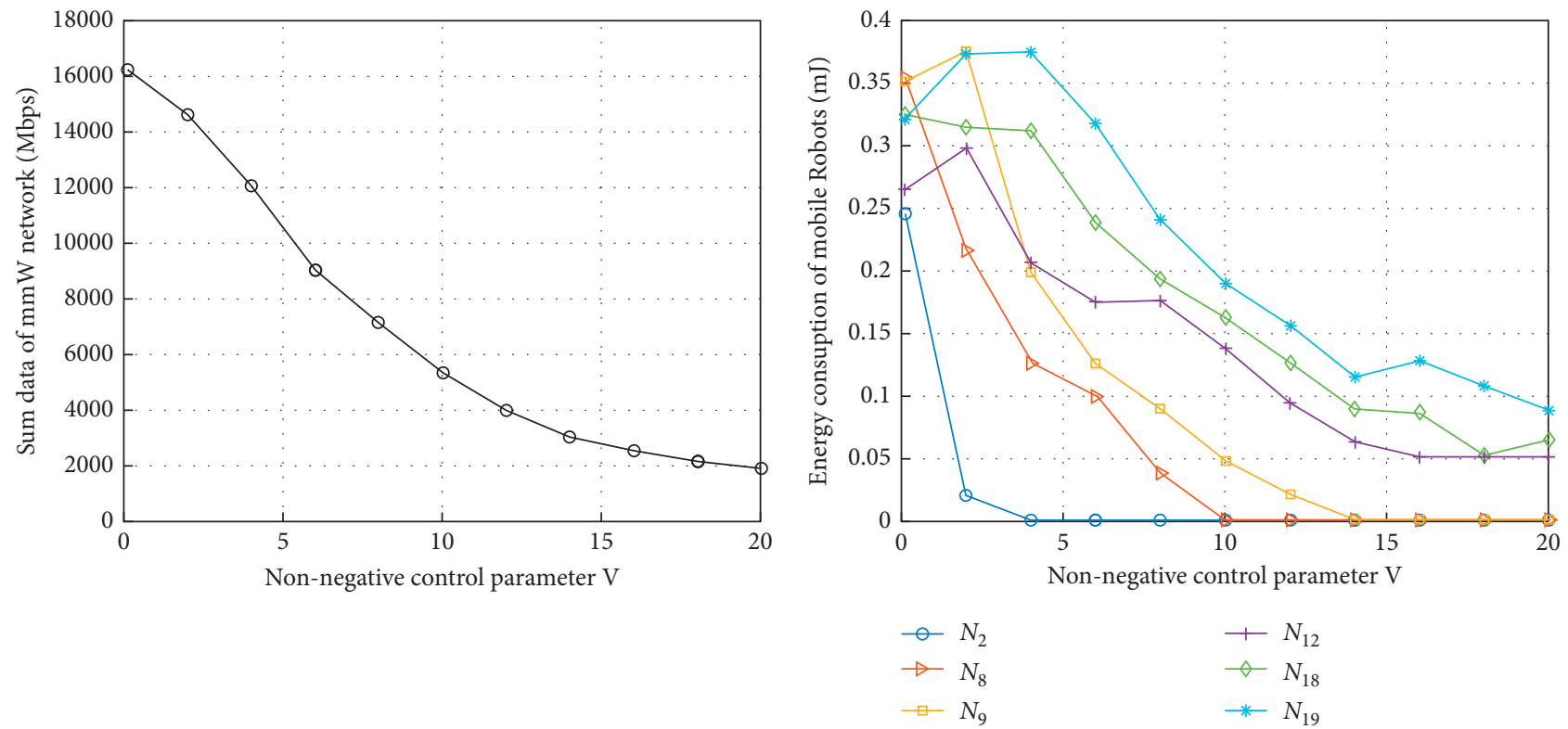

(a)

(b)

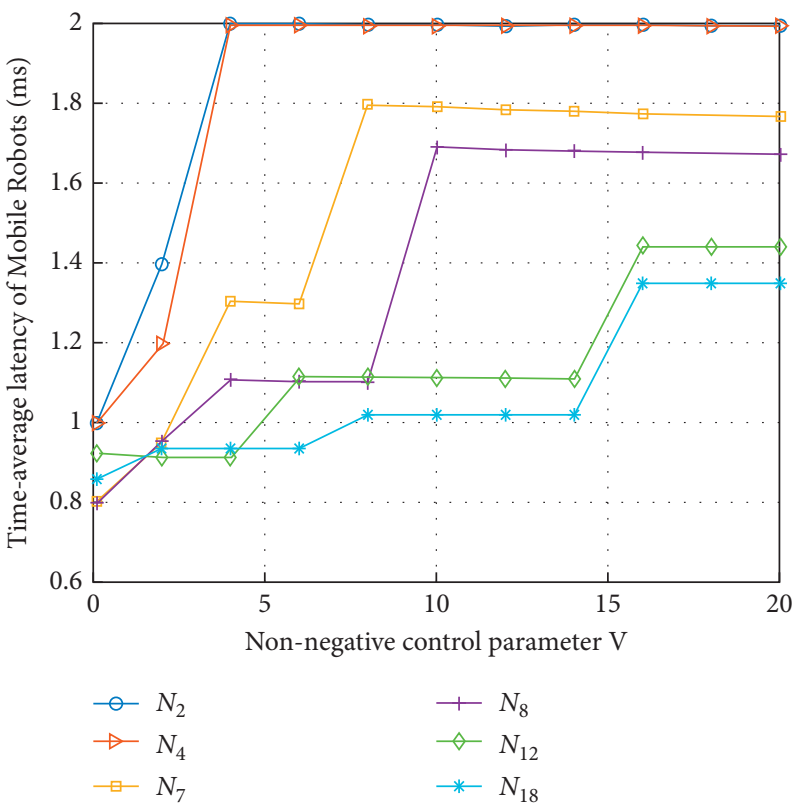

(c)

FIGURE 4: The effects of nonnegative control parameter on mmW network performance: (a) data rate; (b) energy consumption; (c) average latency.

undirected and connected to guarantee that any agent's information can reach any other agents.

According to the above, the Laplacian matrix can be defined as $\mathbf{L}=\mathbf{D e} \mathbf{g}-\mathbf{A}$, where $\mathbf{A}=\left[a_{k n}\right]$ is the adjacency matrix of graph $\mathscr{G}$. We denote $a_{k n}=1$ if $n$ belongs to agent $k$ 's neighbor set, and $a_{k n}=0$ otherwise. De $\mathbf{g}=\operatorname{diag}$ $\left(\sum_{n=1}^{I} a_{1 n}, \ldots, \sum_{n=1}^{I} a_{I n}\right)$ is the degree matrix. Constraint (21a) is defined to guarantee $\lambda_{1}=\lambda_{2}=\cdots=\lambda_{I}$, which insure the consensus among agents $i$ is reached.

Based on the above conversion, the original distributed optimization problem has been decoupled and each agent can decide its own resource and power allocation strategy.
By introducing the Lagrangian multiplier $\mathbf{Z}=\mathrm{col}$ $\left(\mathbf{z}_{1}, \ldots, \mathbf{z}_{I}\right)$, we denote the augmented Lagrangian duality of (20) as

$$
\begin{aligned}
\min _{\mathbf{Z}} \max _{\boldsymbol{\Lambda}} Q(\boldsymbol{\Lambda}, \mathbf{Z})= & \sum_{i \in \mathcal{F}} q_{i}\left(\boldsymbol{\lambda}_{i}\right)-\mathbf{Z}^{T}\left(\mathbf{L} \otimes \mathbf{I}_{i}\right) \boldsymbol{\Lambda} \\
& -\frac{1}{2} \boldsymbol{\Lambda}^{T}\left(\mathbf{L} \otimes \mathbf{I}_{i}\right) \boldsymbol{\Lambda} .
\end{aligned}
$$

According to [19], (22) can be solved by applying the gradient flow and the distributed algorithm for agent $i$ based on projection dynamics given in Algorithm 1 . 
In Algorithm 1, $\dot{f}(\cdot)$ represents gradient calculation of $f$, $\ell$ is the update index, $P_{\Omega_{i}}(x)=\operatorname{argmin}_{y \in \Omega_{i}}\|x-y\|$ is the projection operation of $x$ onto the closed convex set $\Omega_{i}, \nabla f$ means the gradient of $f$, and $\mathcal{N}_{i}$ is the neighbor set of agent $i$. Based on the distributed algorithm, both the power allocation and multiplier parameters can be updated by

$$
\begin{aligned}
\mathbf{p}_{i}^{\mathbf{a}}(\ell+1) & =\mathbf{p}_{i}^{\mathbf{a}}(\ell)+\alpha \mathbf{p}_{i}^{\mathbf{a}}(\ell+1), \\
\lambda_{i}(\ell+1) & =\boldsymbol{\lambda}_{i}(\ell)+\alpha \boldsymbol{\lambda}_{i}(\ell+1), \\
\mathbf{z}_{i}(\ell+1) & =\mathbf{z}_{i}(\ell)+\alpha \dot{\mathbf{z}_{i}}(\ell+1),
\end{aligned}
$$

where $\alpha$ is the integration step.

Algorithm 2 summarizes the detailed procedures of the semidistributed scheme. Specifically, mmW APs achieve the information of Lagrangian multipliers from the information cloud, then calculatetheir power allocation strategy independently, and feed back $\lambda_{i}(\ell)$ and $\mathbf{z}_{i}(\ell), \forall i \in \mathcal{F}$, to the information cloud.

\subsection{The Performance Analysis of the Semidistributed Scheme.} The first and most important advantage of Algorithm 2 is that it does not sacrifice the optimality. We can obtain the global optimal solution for the distributed mmW networks to maximize the utilization efficiency of wireless resource. We introduce the following theorem to prove.

Theorem 2. The proposed semidistributed algorithm can converge to an optimal power allocation solution.

Proof. See Appendix C.

The second merit is initialization-free, which means that the semidistributed algorithm will converge to the global optimal solution under any initial condition due to the projection operation.

\section{Simulation and Evaluation}

In this section, different contrast graphs were obtained by adjusting various parameters. There are multiple line segments with different parameters in each experimental result image. We draw images with lines of different colors and shapes for easy differentiation [5]. Under the coverage of IC, we set 4 $\mathrm{mmW}$ APs, each equipped with $6 \mathrm{RF}$ chains. And there are 24 densely deployed MRs served by the mmW network. We denote the set of MRs as $\mathcal{N}=\left\{N_{1}, N_{2}, \ldots, N_{24}\right\}$, where the minimum data rate of the MRs is set from $0.5 \mathrm{Mbps}$ to 30 Mbps. The remaining parameters are shown in Table 1.

Figure 3 shows the convergence of Algorithm 2 and the effect of integration step on the convergence rate. From the figure, we can observe that the semidistributed power allocation algorithm converges to the optimal solution through iteration, and the larger the integration step setting is, the faster the convergence rate will be. Therefore, we select the appropriate integration step on the premise of ensuring the performance of the algorithm.

Figure 4 shows the variation of data rate, energy consumption, and average latency with the nonnegative control

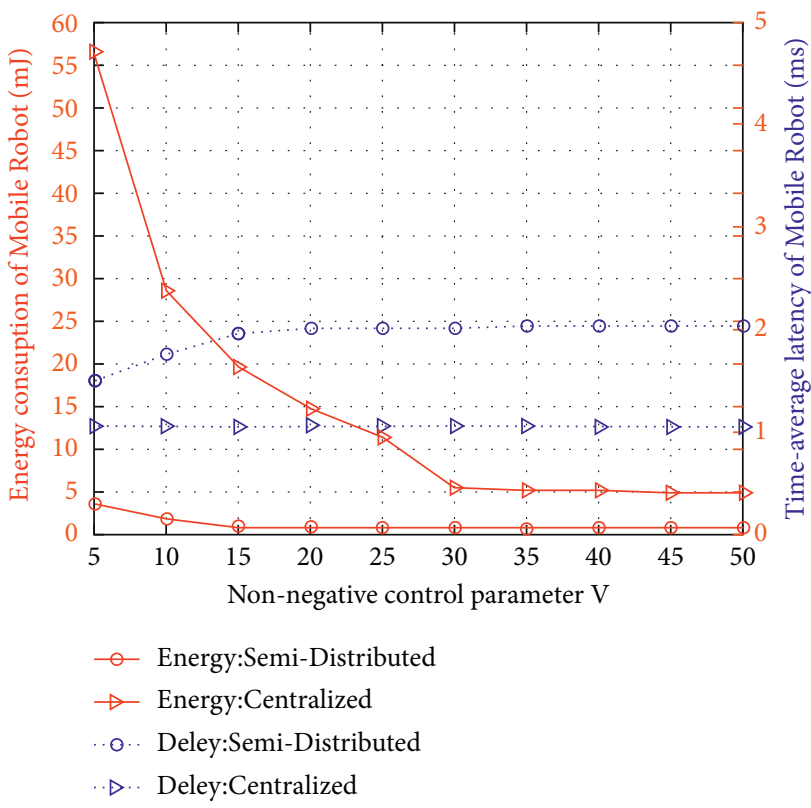

Figure 5: The performance between two different solutions.

parameters $V$, which represents the weight of the objective function in the conversion of Lyapunov drift penalty. From Figure $4(\mathrm{C})$, we can clearly observe that the time-average latency increases gradually with the gradual increase of parameter $V$. In addition, mobile robots with higher data rates usually result in higher energy consumption and lower time latency.

Figure 5 shows the performance of two different solutions. The semidistributed method refers to the proposed resource and power allocation scheme in this paper, and the centralized method refers to solve optimization problems directly. From the picture, we can observe that the semidistributed method has significantly lower energy consumption but slightly higher time-average latency than the centralized method.

\section{Conclusion}

This paper proposes a $\mathrm{mmW}$ communication network for distributed MRs in a MEC environment. While minimizing network energy consumption, a joint resource and power distribution scheme is designed. First, Lyapunov optimization is processed by the target function as a single frame problem and then introduced into the semidistributed algorithm which further solves distributed optimization issues on $\mathrm{mmW}$ MECs. Experiments prove that the program can effectively reduce network energy consumption, and the semidistributed algorithm can greatly reduce signaling overheads, prevent privacy leakage, and further enhance the performance of distributed MRs communication networks.

\section{Appendix}

\section{$\mathbf{A}$}

Proof. of Lemma 1.

Denote $\quad \Delta Z_{j}(t)=\mathbb{E}\left[(1 / 2) Z_{j}(t+1)^{2}-(1 / 2) Z_{j}(t)^{2}\right.$

$\left.\mid Z_{j}(t)\right]$. Without expectation, $\Delta Z_{j}(t)$ can be written as 


$$
\Delta Z_{j}(t)=\frac{1}{2} Z_{j}(t+1)^{2}-\frac{1}{2} Z_{j}(t)^{2}, \quad \forall j \in \mathscr{J} .
$$

Further, we can derive that

$$
\begin{aligned}
& \Delta Z_{j}(t)^{(a)} \leq \frac{1}{2}\left[Q_{j}^{2}(t+1)+2 Q_{j}(t+1) Z_{j}(t)+\left(\bar{Q}_{j}\right)^{2}\right. \\
& \left.\quad-2 \bar{Q}_{j}\left(Q_{j}(t+1)+Z_{j}(t)\right)\right], \\
& \quad{ }^{(b)} 1 \frac{1}{2}\left[Q_{j}^{2}(t)+\left(\bar{Q}_{j}\right)^{2}+\left(R_{j}^{a}(t) \tau\right)^{2}-2 \tau Q_{j}(t) R_{j}^{a}(t)\right. \\
& \left.\quad+\left(A_{j}(t) \tau\right)^{2}+2 \tau Q_{j}(t) A_{j}(t)++2 Q_{j}(t+1) Z j(t)\right], \\
& \quad{ }^{(c)} \frac{1}{2}\left[2 Q_{j}^{2}(t)+\left(\bar{Q}_{j}\right)^{2}-2 \tau Q_{j}(t) R_{j}^{a}(t)+\left(A_{j}(t) \tau\right)^{2}\right. \\
& \left.\quad+2 \tau Q_{j}(t) A_{j}(t)+2 Z_{j}(t)\left(Q_{j}(t)-R_{j}^{a}(t) \tau+A_{j}(t) \tau\right)\right] \\
& \quad \leq \frac{1}{2}\left(Z_{j 1}+Z_{j 2}\right)-R_{j}^{a}(t) \tau\left(Q_{j}(t)+Z_{j}(t)\right), \quad \forall j \in \mathcal{J} .
\end{aligned}
$$

Here, $(a)$ is with the fact of $\left([x]^{+}\right)^{2} \leq x^{2}$, and $(b)$ is proved by the following equation:

$$
\begin{aligned}
& Q_{j}^{2}(t+1)=\left(\left[Q_{j}(t)-R_{j}^{a}(t) \tau\right]^{+}+A_{j}(t) \tau\right)^{2} \\
& \leq\left(\left[Q_{j}(t)-R_{j}^{a}(t) \tau\right]^{+}\right)^{2}+\left(A_{j}(t) \tau\right)^{2} \\
& \quad+2 A_{j}(t) \tau Q_{j}(t) .
\end{aligned}
$$

We assume that the queue data that have not been backlogged and need not to be transmitted; inequation (c) has the causality constraint of

$$
R_{j}^{a}(t) \tau \leq Q_{j}(t)<+\infty, \quad \forall j \in \mathscr{J} .
$$

According to [18], constraint (A.4) can be ignored. Substituting (A.2) into (11), we can obtain the upper bound of $\Delta_{v} \mathbf{Z}(t)$, which ends the proof.

\section{B}

Proof. of Theorem 1.

Substituting (11) into (12), summing up time slot $t$ from 0 to $T-1$, and dividing $T$, we have

$$
\begin{aligned}
& \frac{1}{T} \mathbb{E}\left[\frac{1}{2} \sum_{t=0}^{T-1} \sum_{j \in \mathcal{F}}\left(Z_{j}^{2}(t+1)-Z_{j}^{2}(t)\right)\right]+V \frac{1}{T} \sum_{t=0}^{T-1} \mathbb{E}\left[\sum_{i \in \mathscr{F}} p_{i}^{a}(t) \tau\right] \\
&= \frac{1}{T} \mathbb{E}\left[\frac{1}{2} \sum_{j \in \mathcal{F}}\left(Z_{j}^{2}(T)-Z_{j}^{2}(0)\right)\right]+V \frac{1}{T} \sum_{t=0}^{T-1} \mathbb{E}\left[\sum_{i \in \mathscr{F}} p_{i}^{a}(t) \tau\right] \\
& \stackrel{(a)}{=} \frac{1}{T} \mathbb{E}\left[\sum_{j \in \mathcal{F}} \frac{Z_{j}(T)^{2}}{2}\right]+V \frac{1}{T} \sum_{t=0}^{T-1} \mathbb{E}\left[\sum_{i \in \mathcal{F}} p_{i}^{a}(t) \tau\right] \\
& \stackrel{(b)}{=} \frac{1}{T} \mathbb{E}[L(Z(T))]+V \frac{1}{T} \sum_{t=0}^{T-1} \mathbb{E}\left[\sum_{i \in \mathscr{F}} p_{i}^{a}(t) \tau\right] \\
& \leq \sum_{i \in \mathcal{F}} \frac{Z_{j 1}+Z_{j 2}}{2}+V E(@) \\
& \quad-\frac{1}{T} \sum_{t=0}^{T-1} \mathbb{E}\left[\sum_{j \in \mathcal{F}}\left(Q_{j}(t)+Z_{j}(t)\right) R_{j}^{a}(t) \tau \mid \mathbf{Z}(t)\right],
\end{aligned}
$$

where $(a)$ is due to the assumption of $\mathbf{Z}(0)=0$, and $(b)$ is based on (9). Moreover, $E(\varpi)$ is an arbitrary solution from the Lyapunov function. Let $T \longrightarrow+\infty$, (B.1) can be rewritten as

$$
\begin{aligned}
\lim _{T \longrightarrow+\infty} & \frac{1}{T} \mathbb{E}[L(\mathbf{Z}(T))]+V \lim _{T \longrightarrow+\infty} \frac{1}{T} \sum_{t=0}^{T-1} \mathbb{E}\left[\sum_{i \in \mathscr{F}} p_{i}^{a}(t) \tau\right] \\
& \leq \sum_{i \in \mathscr{F}} \frac{Z_{j 1}+Z_{j 2}}{2}+V E(\Phi) \\
& -\lim _{T \rightarrow+\infty} \frac{1}{T} \sum_{t=0}^{T-1} \mathbb{E}\left[\sum_{j \in \mathscr{F}}\left(Q_{j}(t)+Z_{j}(t)\right) R_{j}^{a}(t) \tau \mid \mathbf{Z}(t)\right],
\end{aligned}
$$

As a stable network, it satisfieslim $\operatorname{li}_{T \rightarrow+\infty}(L(Z(T)) / T)=0$. Hence, (B.2) can be reduced to

$$
\begin{aligned}
S^{\beta} & =\lim _{T \longrightarrow+\infty} \frac{1}{T} \sum_{t=0}^{T-1} \mathbb{E}\left[\sum_{i \in \mathcal{F}} p_{i}^{a}(t) \tau\right] \leq \sum_{j \in \mathcal{F}} \frac{Z_{j 1}+Z_{j 2}}{2 V}+E(\varpi) \\
& -\lim _{T \longrightarrow+\infty} \frac{1}{T V} \sum_{t=0}^{T-1} \mathbb{E}\left[\sum_{j \in \mathcal{F}}\left(Q_{j}(t)+Z_{j}(t)\right) R_{j}^{a}(t) \tau \mid \mathbf{Z}(t)\right] \\
& \leq \sum_{j \in \mathcal{F}} \frac{Z_{j 1}+Z_{j 2}}{2 V}+E(\varpi) .
\end{aligned}
$$


Thereafter, let $\varpi \longrightarrow \Phi^{*}$, we can obtain

$$
S^{\beta} \leq \sum_{j \in \mathcal{F}} \frac{Z_{j 1}+Z_{j 2}}{2 V}+S^{\alpha} .
$$

This ends the proof of (14).

\section{C}

Proof. of Theorem 2.

Without loss of generation, we assume that each agent has only one variable, i.e., $\mathbf{X}_{k} \in \mathbf{R}$. A new vector $\mathbf{S}=\operatorname{col}(\mathbf{X}, \Lambda, \mathbf{Z})$ is defined, and its feasible region is $\bar{\Omega}=\Omega \times \mathbf{R}^{\mathbf{K}} \times \mathbf{R}^{\mathbf{K}}$. By applying the gradient flow, the updating rules of all agents can be expressed as

$$
\dot{S}=\mathbf{P}_{\bar{\Omega}}(\mathbf{S}-\mathbf{F}(\mathbf{S}))-\mathbf{S}
$$

where the vector function $F(\mathbf{S})$ is denoted as

$$
F(\mathbf{S})=\left(\begin{array}{c}
\nabla f(\mathbf{X})-\mathbf{G} \boldsymbol{\Lambda} \\
\mathbf{L} \boldsymbol{\Lambda}+\mathbf{L Z}-(\mathbf{I}-\mathbf{G X}) \\
-\mathbf{L} \boldsymbol{\Lambda}
\end{array}\right) .
$$

To prove the convergence of the proposed algorithm, the Lyapunov stability can be applied. First, we introduce a Lyapunov function to analyze the Lyapunov stability, which is given by

$$
V_{g}=-\langle F(\mathbf{S}), H(\mathbf{S})-\mathbf{S}\rangle-\frac{1}{2}\|H(\mathbf{S})-\mathbf{S}\|_{2}^{2}+\frac{1}{2}\left\|\mathbf{S}-\mathbf{S}^{*}\right\|_{2}^{2},
$$

where $H(\mathbf{S})=P_{\bar{\Omega}}(\mathbf{S}-F(\mathbf{S})), \mathbf{S}^{*}=\operatorname{col}\left(\mathbf{X}^{*}, \Lambda^{*}, \mathbf{Z}^{*}\right), \mathbf{X}^{*}$ is the optimal solution, and $\Lambda^{*}$ and $Z^{*}$ are the optimal multipliers. To guarantee the Lyapunov stability of the equilibrium point, $V_{g}$ must be constant positive or constant negative. Due to

$$
\begin{aligned}
& -\langle F(\mathbf{S}), H(\mathbf{S})-\mathbf{S}\rangle-\frac{1}{2}\langle H(\mathbf{S})-\mathbf{S}, H(\mathbf{S})-\mathbf{S}\rangle \\
& \geq \frac{1}{2}\|\mathbf{S}-H(\mathbf{S})\|_{2}^{2},
\end{aligned}
$$

we can obtain that $V_{g} \geq(1 / 2)\|\mathbf{S}-H(\mathbf{S})\|_{2}^{2}+(1 / 2)\left\|\mathbf{S}-\mathbf{S}^{*}\right\| \geq 0$ and $V_{q}=0$ if and only if $\mathbf{S}=\mathbf{S}^{*}$.

Since any asymmetric variational inequality can be converted to a differentiable optimization problem [21], $V_{g}$ can be expressed as

$$
\begin{aligned}
& \dot{V}_{g}=\left(F(\mathbf{S})-\left(J_{F}(\mathbf{S})-I\right)(H(\mathbf{S})-\mathbf{S})+\mathbf{S}-\mathbf{S}^{*}\right)^{T} \\
& \quad(H(\mathbf{S})-\mathbf{S}),
\end{aligned}
$$

where $J_{F}(\mathbf{S})$ is the Jacobian matrix of $F(\mathbf{S})$, which is defined as

$$
J_{F}(\mathbf{S})=\left(\begin{array}{ccc}
\nabla^{2} R(\mathbf{X}) & -\mathbf{D} & 0 \\
\mathbf{D} & \mathbf{L} & \mathbf{L} \\
0 & -\mathbf{L} & 0
\end{array}\right) .
$$

According to the basic property of projection operation, it can be given that

$$
\left\langle\mathbf{S}-F(\mathbf{S})-H(\mathbf{S}), H(\mathbf{S})-\mathbf{S}^{*}\right\rangle \geq 0,
$$

which is equivalent to

$$
\left\langle\mathbf{S}-F(\mathbf{S})-H(\mathbf{S}), H(\mathbf{S})-\mathbf{S}+\mathbf{S}-\mathbf{S}^{*}\right\rangle \geq 0 .
$$

Then, we can infer

$$
\begin{aligned}
& \left\langle\mathbf{S}-\mathbf{S}^{*}+F(\mathbf{S}), H(\mathbf{S})-\mathbf{S}\right\rangle+\|H(\mathbf{S})-\mathbf{S}\|_{2}^{2} \\
& \leq-\left\langle F(\mathbf{S}), \mathbf{S}-\mathbf{S}^{*}\right\rangle .
\end{aligned}
$$

With the assumption that $R\left(\mathbf{X}_{k}\right)$ are strictly convex and the information exchanging graph is undirected and connected, the following result can be obtained:

$$
\mathbf{S}^{T} J_{F}(\overline{\mathbf{S}}) \mathbf{S}=\mathbf{X}^{T} \nabla^{2} R(\overline{\mathbf{X}}) \mathbf{X}+\boldsymbol{\Lambda}^{T} \mathbf{L} \boldsymbol{\Lambda}>0, \quad \forall \overline{\mathbf{S}} \in \overline{\mathbf{\Omega}} .
$$

Based on (C.9) and (C.10), $\dot{V}_{g}$ can be further analyzed as follows:

$$
\begin{aligned}
\dot{V}_{g}= & -(H(\mathbf{S})-\mathbf{S})^{T} J_{F}(\mathbf{S})(H(\mathbf{S})-\mathbf{S})+\|H(\mathbf{S})-\mathbf{S}\|_{2}^{2} \\
& +\left\langle\mathbf{S}-\mathbf{S}^{*}+F(\mathbf{S}), H(\mathbf{S})-\mathbf{S}\right\rangle \\
& \leq-\left\langle F(\mathbf{S}), \mathbf{S}-\mathbf{S}^{*}\right\rangle .
\end{aligned}
$$

Since $\left\langle F(\mathbf{S}), \mathbf{S}-\mathbf{S}^{*}\right\rangle \geq 0, \dot{V}_{g} \leq 0$, and the equilibrium point is assumed to be $\mathbf{S}^{*}$. Therefore, the equilibrium point is Lyapunov stable. Hence, the proposed distributed algorithm can converge to its equilibrium point, which ends the proof.

\section{Data Availability}

Data sharing is not applicable to this article as no datasets were generated or analyzed during the current study.

\section{Conflicts of Interest}

The authors declare that they have no conflicts of interest.

\section{References}

[1] Y. Zhang, Q. Xia, and Y. Xu, "Multi-robot cooperation system based on wireless network," in Proceedings of the 2019 IEEE SmartWorld, Ubiquitous Intelligence Computing, Advanced 
Trusted Computing, Scalable Computing Communications, Cloud Big Data Computing, Internet of People and Smart City Innovation (SmartWorld/SCALCOM/UIC/ATC/CBDCom/ IOP/SCI), pp. 162-165, Leicester, UK, February 2019.

[2] Y. Li, F. Du, P. Wang, and Y. Yin, "A multi-robot topic communication method based on tcp and udp," in Proceedings of the 2020 Chinese Automation Congress (CAC), pp. 668-672, Shanghai, China, November 2020.

[3] J. Karjee, S. Behera, H. Kumar Rath, and A. Simha, "Distributed cooperative communication and link prediction in cloud robotics,", in Proceedings of the 2017 IEEE International Conference on Sensing, Communication and Networking (SECON Workshops), pp. 1-7, San Diego, CA, USA, June 2017.

[4] M. Fu, S. Sun, K. Ni, and X. Hou, "Mobile robot object recognition in the internet of things based on fog computing," in Proceedings of the 2019 Asia-Pacific Signal and Information Processing Association Annual Summit and Conference (APSIPA ASC), pp. 1838-1842, Lanzhou, China, November 2019.

[5] F. Ahmed, A. A. Dowhuszko, and O. Tirkkonen, "Distributed algorithm for downlink resource allocation in multicarrier small cell networks," in Proceedings of the 2012 IEEE International Conference on Communications (ICC), pp. 68026808, Ottawa, ON, Canada, June 2012.

[6] J. Xiao, C. Yang, A. Anpalagan, Q. Ni, and M. Guizani, "Joint interference management in ultra-dense small-cell networks: a multi-domain coordination perspective," IEEE Transactions on Communications, vol. 66, no. 11, pp. 5470-5481, 2018.

[7] M. Chen, W. Saad, and C. Yin, "Echo state networks for selforganizing resource allocation in LTE-U with uplinkdownlink decoupling," IEEE Transactions on Wireless Communications, vol. 16, no. 1, pp. 3-16, 2017.

[8] R. Yin, S. Liu, G. Yu, Y. Zhang, and Q. Chen, "Semi-distributed joint power and spectrum allocation for laa based small cell networks," IEEE Transactions on Wireless Communications, vol. 19, no. 6, pp. 4141-4153, 2020.

[9] F. Vhora and J. Gandhi, "A comprehensive survey on mobile edge computing: challenges, tools, applications," in Proceedings of the 2020 Fourth International Conference on Computing Methodologies and Communication (ICCMC), pp. 49-55, Erode, India, April 2020.

[10] M. Satyanarayanan, "The emergence of edge computing," Computer, vol. 50, no. 1, pp. 30-39, 2017.

[11] M. McClellan, C. Cervelló-Pastor, and S. Sallent, "Deep learning at the mobile edge: opportunities for $5 \mathrm{~g}$ networks," Applied Sciences, vol. 10, no. 14, p. 4735, 2020.

[12] D. Wu, J. Wang, Y. Cai, and M. Guizani, "Millimeter-wave multimedia communications: challenges, methodology, and applications," IEEE Communications Magazine, vol. 53, no. 1, pp. 232-238, 2015.

[13] S. Barbarossa, E. Ceci, M. Merluzzi, and E. Calvanese-Strinati, "Enabling effective mobile edge computing using millimeterwave links," in Proceedings of the 2017 IEEE International Conference on Communications Workshops (ICC Workshops), pp. 367-372, Paris, France, May 2017.

[14] F. Guo, L. Ma, H. Zhang, H. Ji, and X. Li, "Joint load management and resource allocation in the energy harvesting powered small cell networks with mobile edge computing," in Proceedings of the IEEE INFOCOM 2018 - IEEE Conference on Computer Communications Workshops (INFOCOMWKSHPS), pp. 299-304, Piscataway, NJ, USA, April 2018.
[15] Q. Han, G. Zheng, and C. Xu, "Energy-efficient resource allocation for mobile edge computing in noma-enabled small cell networks," in Proceedings of the 2020 IEEE 20th International Conference on Communication Technology (ICCT), pp. 415-419, China, Nanning, October 2020.

[16] H. Shokri-Ghadikolaei, C. Fischione, G. Fodor, P. Popovski, and M. Zorzi, "Millimeter wave cellular networks: a mac layer perspective," IEEE Transactions on Communications, vol. 63, no. 10, pp. 3437-3458, 2015.

[17] Q. Chen, X. Xu, H. Jiang, and X. Liu, "An energy-aware approach for industrial internet of things in $5 \mathrm{~g}$ pervasive edge computing environment," IEEE Transactions on Industrial Informatics, vol. 17, no. 7, pp. 5087-5097, 2021.

[18] M. J. Neely, "A lyapunov optimization approach to repeated stochastic games," in Proceedings of the 2013 51st Annual Allerton Conference on Communication, Control, and Computing (Allerton), pp. 1082-1089, Monticello, IL, USA, October 2013.

[19] P. Yi, Y. Hong, and F. Liu, "Initialization-free distributed algorithms for optimal resource allocation with feasibility constraints and its application to economic dispatch of power systems," Automatica, vol. 74, 2015.

[20] M. Mesbahi and M. Egerstedt, "Graph theoretic methods in multiagent networks," Princeton University Press, vol. 33, 2010.

[21] M. Fukushima, "Equivalent differentiable optimization problems and descent methods for asymmetric variational inequality problems," Mathematical Programming, vol. 53, no. 1, pp. 99-110, 1992. 